\title{
Analogies and disparities among scintigraphic bone tracers in the diagnosis of cardiac and non- cardiac ATTR amyloidosis
}

\author{
Claudio Rapezzi, MD, ${ }^{\mathrm{a}, \mathrm{b}}$ Christian Gagliardi, $M D,{ }^{\mathrm{a}, \mathrm{b}}$ and Agnese Milandri, $\mathrm{MD}^{\mathrm{a}, \mathrm{b}}$ \\ a Cardiology, DIMES, Alma Mater University of Bologna, Bologna, Italy \\ b Policlinico S.Orsola-Malpighi, Bologna, Italy
}

Received Feb 6, 2018; accepted Feb 6, 2018

doi: $10.1007 / \mathrm{s} 12350-018-1235-6$

In this issue of JNC, BW Spery and Coll report a retrospective analysis of 57 patients with transthyretin-related amyloidosis (ATTR) in an advanced phase of the disease who underwent 99mTechnetium-pyrophosphate (99mTcPYP) scintigraphy. Although relatively small and "negative," the study is relevant since it broadens our knowledge on the uptake of "bone tracers" in ATTR and contributes to understand the limitations of the clinical use of scintigraphy in this disease. The paper raises, directly or indirectly, at least three questions: To what extent are the different bone tracers interchangeable for the diagnosis of ATTR cardiac amyloidosis? Are bone tracers able to image non-cardiac ATTR amyloidosis? What is the explanation for the variable performance of the different bone tracers in the diagnosis of cardiac and extracardiac ATTR amyloidosis?

Key Words: Cardiomyopathy $\cdot$ Technetium-99m $\cdot$ Diagnostic and prognostic application

\section{See related article, pp. 1630-1637}

In this issue of JNC, BW Spery and Coll report a retrospective analysis of 57 patients with transthyretinrelated amyloidosis (ATTR) in an advanced phase of the disease who underwent $99 \mathrm{mTechnetium-pyrophosphate}$ (99mTcPYP) scintigraphy. ${ }^{1}$ The study was aimed to investigate the skeletal muscle uptake of the tracer. In fact, in previous scintigraphic studies with 99mTechnetium-3,3-diphosphono-1,2-propanodicarboxylic acid (99mTcDPD), skeletal muscle uptake had been reported and had even been considered a potential source of attenuation of the bony uptake. ${ }^{2}$ Spery and Coll found that skeletal muscle uptake of $99 \mathrm{mTcPYP}$ was minimal when assessed by qualitative and quantitative metrics and

Reprint requests: Claudio Rapezzi, MD, Policlinico S.Orsola-Malpighi, Via Massarenti 9, 40138 Bologna, Italy; claudio.rapezzi@unibo.it J Nucl Cardiol 2019;26:1638-41.

1071-3581/ $\$ 34.00$

Copyright (c) 2018 American Society of Nuclear Cardiology. was not significantly different in patients with Grade 2 vs 3 myocardial uptake. Hence, they conclude that the properties of 99mTcPYP may be different than 99mTcDPD with respect to non-cardiac uptake and that 99mTcPYP cannot be used to image extracardiac ATTR.

Although relatively small and "negative," the study is relevant since it broadens our knowledge on the uptake of "bone tracers", in ATTR and contributes to understand the limitations of the clinical use of scintigraphy in this disease.

The paper raises, directly or indirectly, at least three questions:

(1) To what extent are the different bone tracers interchangeable for the diagnosis of cardiac ATTR?

(2) Are bone tracers able to image non-cardiac ATTR?

(3) What is the explanation for the variable performance of the different bone tracers in the diagnosis of cardiac and extracardiac ATTR?

\section{INTERCHANGEABILITY OF THE DIFFERENT TRACERS FOR THE IMAGING OF CARDIAC ATTR}

The 99m-Technetium (99mTc) bisphosphonate derivatives, originally developed for bone imaging, 
have found a second life as a non-invasive diagnostic tool for cardiac ATTR. ${ }^{3}$ Three tracers have been extensively studied in different countries: $99 \mathrm{mT}$ TPYP is readily available in USA since it has been approved by FDA; 99mTcDPD and 99mTechnetium hydroxydiphosphonate (99mTcHMDP), which has a chemical composition similar to that of $99 \mathrm{mTcPYP}$, have been examined in many European single-center studies. The most common $99 \mathrm{mTc}$ bone scintigraphy techniques include injection of 10 to $20 \mathrm{mCi}$ of $99 \mathrm{mTc}$ PYP, DPD, or HMDP; imaging at $60 \mathrm{~min}$ to $3 \mathrm{~h}$; planar imaging followed by single-photon emission computed tomography (SPECT) if results are positive; and possibly delayed whole-body imaging. Diffuse myocardial uptake of $99 \mathrm{mTc}$ radiotracers is graded according to a standardized system (0, no myocardial uptake; 1, myocardial uptake less than bone; 2 , myocardial uptake equal to bone; 3 , myocardial uptake greater than bone). ${ }^{4}$

Studies on the diagnostic accuracy of the single tracers in the recognition of cardiac ATTR are relatively numerous, while head to head comparisons between tracers are very limited.

\section{Studies on the Diagnostic Performance of the Single Bone Tracers}

Although 99mTcPYP was firstly described to accumulate in the heart of patients with cardiac amyloidosis, in the early 1980s, the role of bone tracers scintigraphy in the diagnosis of cardiac amyloidosis (CA) has been systematically studied only after 2000s and the attention was initially focused on 99mTcDPD. ${ }^{4-9}$ Perugini and Coll firstly documented that a visual score $\geq 1$ had $100 \%$ sensitivity and $100 \%$ specificity in identifying ATTR-CA. ${ }^{4}$ In a subsequent study in a wider cohort of patients, the same group, ${ }^{7}$ using score $\geq 2$ as diagnostic criteria, actually confirmed a very high sensitivity $(100 \%)$, but a lower specificity (82\%), mainly due to the presence of mild and moderate cardiac uptake in light chain amyloidosis (AL)-CA. A visual score $\geq 2$ also allowed to differentiate wild-type (wt)-ATTR-CA from other causes of hypertrophic cardiomyopathy in a group of 62 patients with $100 \%$ specificity and sensibility. $^{9}$

The important role of bone scintigraphy in the etiological diagnosis of $\mathrm{CA}$ has been subsequently confirmed with $99 \mathrm{mTcPYP}^{10-13}$ and $99 \mathrm{mTcHMDP} .^{14-18}$ On the contrary, 99mTc-methylene diphosphonate (99mTcMDP) is currently considered inappropriate as a tracer for the non-invasive diagnosis of ATTR-CA since it failed to show myocardial uptake in the majority of the (few) dedicated studies. ${ }^{5,8,19-21}$ The diagnostic role of bone tracers in ATTR has been recently reviewed by an international group of cardiac amyloid experts. ${ }^{3}$ In a cumulative analysis of 1217 patients (857 with histologically proven amyloidosis and 360 with nonamyloid cardiomyopathy) who underwent $99 \mathrm{mTc}$ bone scintigraphy (99mTcPYP, 99mTcDPD, and 99mTcHMDP) for suspected ATTR-CA, 99mTc bone scintigraphy was highly sensitive at $99 \%$ and was $86 \%$ specific for ATTR-CA (with almost all false-positives from AL-CA). So, this consensus paper concluded that cardiac ATTR can be reliably diagnosed in the absence of histology in patients with an echocardiogram or cardiac magnetic resonance suggestive of amyloidosis, moderate-intense (visual score grade 2 or 3 ) cardiac uptake on a radionuclide scan (with 99mTcDPD, 99mTcPYP, or 99mTcHMDP) and absence of a detectable monoclonal protein in blood and urine tests.

\section{Head to Head Studies}

Although diagnostic accuracies of the different bone tracers in the recognition of ATTR-CA are generally considered equivalent, very few studies have directly compared the tracers head to head. Most of these studies are small and anecdotal and compared 99mTcMDP and other tracers. These results have been constantly disappointing due to the very low sensitivity of $99 \mathrm{mTcMDP}$. In a substudy from our group, aimed to compare 99mTcMDP with 99mTcDPD, for example, none of 11 patients with a positive $99 \mathrm{mTcDPD}$ scintigraphy did uptake $99 \mathrm{mTcMDP}$ at myocardial level. ${ }^{5}$ On the contrary, in the only head to head available study with a positive result, the hydroxymethylated compound 99mTcHMDP has been tested in a direct comparison with $99 \mathrm{mTcDPD}$ in six ATTR patients and turned out to be comparable in terms of myocardial uptake. ${ }^{22}$

\section{IMAGING OF EXTRACARDIAC ATTR WITH BONE TRACERS}

Extracardiac uptake of $99 \mathrm{mTcDPD}$ has been previously shown and has been considered the expression of multiorgan transthyretin (TTR) involvement. ${ }^{2}$ It is well known that many extracardiac tissues and organs, including lung, peripheral muscles, ligamentum carpale, muscular tendons, and abdominal fat can be involved by ATTR. Since the consequences can be relevant in some patients, the availability of non-invasive imaging techniques able to detect this phenomenon is clinically relevant. In a first study published in 2014, Hutt and Coll $^{23}$ noted a consistent and unusual pattern of 99mTcDPD uptake involving the gluteal shoulder chest and abdominal wall regions in 70/77 patients with cardiac ATTR (both wild-type-TTR and mutant-TTR). In a more recent and larger study (563 patients with ATTR-CA) by the same group, the soft tissue-to-femur 
ratio of counts (a plausible marker of extracardiac amyloid infiltration) progressively increased from patients with score 0 to patients with score 3 and in particular from score 2 to 3 . They employed a "standard" imaging protocol with late-phase image acquisition (whole-body planar images acquired $3 \mathrm{~h}$ after tracer injection followed by cardiac SPECT-CT). According to the authors, this finding contributed to the attenuation of the bone signal (the marker of the score 3 ). So, following this interpretation, the difference between grade 2 and 3 does not necessarily reflect per se a different (more advanced) stage of cardiac involvement and this could be one of the reasons why the specific grade of positivity, according to the Perugini classification, provides little prognostic information in ATTR. ${ }^{2}$ More recently, Cappelli and Colleagues ${ }^{24}$ have reported their retrospective experience in the visualization of lung deposition of amyloid using 99mTcHMDP scintigraphy. In a small cohort of 82 patients, lung uptake was present in $60 \%$ of ATTR cases, but only in $6 \%$ patients with AL disease. Notably, the degree of lung and heart retention were interrelated, and the selectivity was the same for both organs in ATTR. Therefore, two of the three bone tracers currently used to detect ATTR cardiac amyloidosis have been shown to image also extracardiac ATTR deposits, whereas $99 \mathrm{mTcPYP}$, according to the present study, would not be able to recognize non-cardiac localizations of ATTR. It appears reasonable to state that the varying ability to image non-cardiac ATTR deposition among bone tracers is due to different properties of the single compounds more than to methodological aspects or different patients' characteristics.

\section{EXPLORING THE MECHANISMS OF HETEROGENEITY OF TRACER'S UPTAKE IN ATTR}

Figure 1 summarizes the most likely determinants of this heterogeneity.

The variable myocardial uptake according to type of amyloidosis (in particular, ATTR vs AL) has been one of the first peculiarities to be noted. ${ }^{5}$ ATTR-CA is particularly avid for bone tracers, whereas uptake in ALCA is either absent or mild. ${ }^{3,7}$ The intimate mechanism for this differential uptake is unknown, but it has been suggested that the preferential binding to ATTR may be a result of higher calcium content. ${ }^{25}$ More in general, the role of calcium within the myocardial tissue appears crucial. Recently, using a special calcium stain as well as immunohistochemistry to investigate the expression of macrophages, Stats and Stone evaluated endomyocardial biopsies of patients with ATTR and AL and found significantly greater density of small microcalcifications in cases of ATTR compared to $\mathrm{AL},{ }^{26}$ although

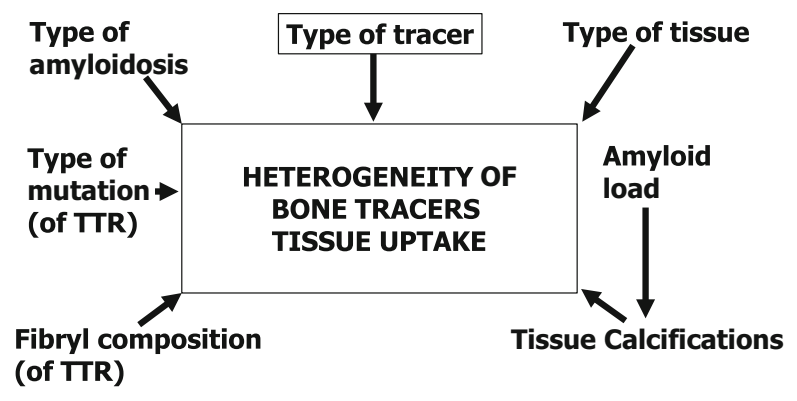

Figure 1. Main possible determinants of heterogeneity in tissue uptake of bone tracers in patients with systemic amyloidosis.

a few cases demonstrated levels of microcalcifications comparable to the ATTR patients. Another recently evaluated tracer, $18 \mathrm{~F}-\mathrm{NaF}$, a bone radiotracer that is used predominantly to detect prostate cancer metastases and microcalcifications within the coronary arteries, has been tested for imaging of ATTR-CA, with some interesting results. ${ }^{27}$

Although the amount of calcium within the infiltrated tissue is likely related to the overall amyloid load which in turn is related with ventricular thickness and mass, the correlation between ventricular parietal thickness and semiquantitative measurements of bone tracer uptake is very weak. ${ }^{2,5,6}$

Recently, the role of other two possible modulators of the binding between amyloid deposits and the bone tracers has been highlighted: the type of mutation and the result of the proteolysis of fibers at myocardial level (full length only vs full length plus C-terminal ATTR fragments). Diagnostic sensitivity of $99 \mathrm{mTcDPD}$ is low for patients with early-onset V30 M mutation. Typically, these patients have ATTR fibrils composed of fulllength TTR (type B fibrils), whereas most other mutations, including late-onset V30M, have fibrils composed of a mixture of full-length and C-terminal fragments of TTR (type A fibrils). ${ }^{28,29}$

Therefore, the different diagnostic performances of bone tracers as imaging tools for cardiac and extracardiac amyloidosis must be considered in a wider context and within a challenging pathophysiologic scenario.

\section{Disclosure} disclose.

The authors declare that there is no conflict of interest to

\section{References}

1. Sperry BW, Gonzalez MH, Brunken R, Cerqueira MD, Hanna M, Jaber WA. Non-cardiac uptake of technetium-99m pyrophosphate 
in transthyretin cardiac amyloidosis. J Nucl Cardiol 2018. https://doi.org/10.1007/s12350-017-1166-7.

2. Hutt DF, Fontana M, Burniston M, Quigley AM, Petrie A, Ross JC, et al. Prognostic utility of the Perugini grading of $99 \mathrm{mTc}$ DPD scintigraphy in transthyretin (ATTR) amyloidosis and its relationship with skeletal muscle and soft tissue amyloid. Eur Heart J Cardiovasc Imaging 2017;18:1344-50.

3. Gillmore JD, Maurer MS, Falk RH, Merlini G, Damy T, Dispenzieri A, et al. Nonbiopsy diagnosis of cardiac transthyretin amyloidosis. Circulation 2016;133(24):2404-12.

4. Puille M, Altland K, Linke RP, Steen-Müller MK, Kiett R, Steiner $\mathrm{D}$, et al. $99 \mathrm{mTc}-\mathrm{DPD}$ scintigraphy in transthyretin-related familial amyloidotic polyneuropathy. Eur J Nucl Med Mol Imaging 2002;29:376-9.

5. Perugini E, Guidalotti PL, Salvi F, Cooke RM, Pettinato C, Riva $\mathrm{L}$, et al. Noninvasive etiologic diagnosis of cardiac amyloidosis using 99mTc-3,3-diphosphono-1,2-propanodicarboxylic acid scintigraphy. J Am Coll Cardiol 2005;46:1076-84.

6. Rapezzi C, Quarta CC, Guidalotti PL, Pettinato C, Fanti S, Leone $\mathrm{O}$, et al. Role of $(99 \mathrm{~m}) \mathrm{Tc}$-DPD scintigraphy in diagnosis and prognosis of hereditary transthyretin-related cardiac amyloidosis. JACC Cardiovasc Imaging 2011;4:659-70.

7. Rapezzi C, Quarta CC, Guidalotti PL, Longhi S, Pettinato C, Leone $\mathrm{O}$, et al. Usefulness and limitations of $99 \mathrm{mTc}-3,3-$ diphosphono-1,2-propanodicarboxylic acid scintigraphy in the aetiological diagnosis of amyloidotic cardiomyopathy. Eur J Nucl Med Mol Imaging 2011;38:470-8.

8. De Haro-del Moral FJ, Sánchez-Lajusticia A, Gómez-Bueno M, García-Pavía P, Salas-Antón C, Segovia-Cubero J. Role of cardiac scintigraphy with 99mTc-DPD in the differentiation of cardiac amyloidosis subtype. Revista Española de Cardiologíap Cardiol (Engl Ed) 2012;65:440-6.

9. Quarta CC, Guidalotti PL, Longhi S, Pettinato C, Leone O, Ferlini A, et al. Defining the diagnosis in echocardiographically suspected senile systemic amyloidosis. JACC Cardiovasc Imaging 2012;5:755-8

10. Yamamoto Y, Onoguchi M, Haramoto M, Kodani N, Komatsu A, Kitagaki H, Tanabe K. Novel method for quantitative evaluation of cardiac amyloidosis using (201) TlCl and (99m)Tc-PYP SPECT. Ann Nucl Med 2012;26:634-43.

11. Bokhari S, Castano A, Pozniakoff T, Deslisle S, Latif F, Maurer MS. (99m)Tc-pyrophosphate scintigraphy for differentiating lightchain cardiac amyloidosis from the transthyretin-related familial and senile cardiac amyloidoses. Circ Cardiovasc Imaging 2013;6:195-201

12. Papantoniou V, Valsamaki P, Kastritis S, Tsiouris S, Delichas Z, Papantoniou Y, Tsiouma M, Athanasoulis T, Fotopoulos A, Dimopoulos MA. Imaging of cardiac amyloidosis by $(99 \mathrm{~m}) \mathrm{Tc}-$ PYP scintigraphy. Hellenic J Nucl Med 2015;18:42-50.

13. Castano A, Haq M, Narotsky DL, Goldsmith J, Weinberg RL, Morgenstern R, et al. Multicenter study of planar technetium $99 \mathrm{~m}$ pyrophosphate cardiac imaging: Predicting survival for patients with ATTR cardiac amyloidosis. JAMA Cardiol 2016;1:880-9.

14. Glaudemans AW, van Rheenen RW, van den Berg MP, Noordzij $\mathrm{W}$, Koole $\mathrm{M}$, Blokzijl $\mathrm{H}$, et al. Bone scintigraphy with 99mTechnetium-hydroxymethylene diphosphonate allows early diagnosis of cardiac involvement in patients with transthyretinderived systemic amyloidosis. Amyloid 2014;21:35-44.

15. Galat A, Rosso J, Guellich A, Van Der Gucht A, Rappeneau S, Bodez D, et al. Usefulness of (99m)Tc-HMDP scintigraphy for the etiologic diagnosis and prognosis of cardiac amyloidosis. Amyloid 2015;22:210-20.

16. Galat A, Van der Gucht A, Guellich A, Bodez D, Cottereau AS, Guendouz S, et al. Early phase ${ }^{99} \mathrm{Tc}-\mathrm{HMDP}$ Scintigraphy for the diagnosis and typing of cardiac amyloidosis. JACC Cardiovasc Imaging 2017;10:601-3.

17. Cappelli F, Gallini C, Di Mario C, Costanzo EN, Vaggelli L, Tutino F, et al. Accuracy of 99mTc-Hydroxymethylene diphosphonate scintigraphy for diagnosis of transthyretin cardiac amyloidosis. J Nucl Cardiol 2017. https://doi.org/10.1007/ s12350-017-0922-z.

18. Van Der Gucht A, Cottereau AS, Abulizi M, Guellich A, BlancDurand P, Israel JM, et al. Apical sparing pattern of left ventricular myocardial ${ }^{99} \mathrm{Tc}-\mathrm{HMDP}$ uptake in patients with transthyretin cardiac amyloidosis. J Nucl Cardiol 2017. https://doi.org/10. 1007/s12350-017-0894-z.

19. Lee VW, Caldarone AG, Falk RH, Rubinow A, Cohen AS. Amyloidosis of heart and liver: comparison of Tc-99m pyrophosphate and Tc-99m methylene diphosphonate for detection. Radiology 1983;148:239-42.

20. Ferreira SG, Rocha AM. Moreira do Nascimento OJ, Mesquita CT. Role of $(99 \mathrm{~m}) \mathrm{Tc}-\mathrm{DPD}$ scintigraphy on discrimination of familial cardiac amyloidosis. Int J Cardiol 2016;203:885-7.

21. Yang JC, Fox J, Chen C, Yu AF. Cardiac ATTR amyloid nuclear imaging - not all bone scintigraphy radionuclide tracers are created equal. J Nucl Cardiol 2017. https://doi.org/10.1007/ s12350-017-1141-3.

22. Abulizi M, Cottereau AS, Guellich A, Vandeventer S, Galat A, Van Der Gucht A, et al. Early-phase myocardial uptake intensity of ${ }^{99} \mathrm{Tc}-\mathrm{HMDP}$ vs ${ }^{99} \mathrm{Tc}$-DPD in patients with hereditary transthyretin-related cardiac amyloidosis. J Nucl Cardiol 2016;25:21722.

23. Hutt DF, Quigley AM, Page J, Hall ML, Burniston M, Gopaul D, et al. Utility and limitations of 3,3-diphosphono-1,2-propanodicarboxylic acid scintigraphy in systemic amyloidosis. Eur Heart $\mathbf{J}$ Cardiovasc Imaging 2014;15:1289-98.

24. Cappelli F, Gallini C, Costanzo EN, Tutino F, Ciaccio A, Vaggelli $\mathrm{L}$, et al. Lung uptake during $99 \mathrm{~m}-\mathrm{Tc}$ hydroxymethylene diphosphonate scintigraphy in patients with TTR cardiac amyloidosis: an underestimated phenomenon. Intern J Cardiol 2017;254:346-50.

25. Willerson JT, Parkey RW, Bowe TJ, Lewis SE, Corbett J, Buja LM. Pathophysiologic considerations and clinicopathological correlates of technetium-99m stannous pyrophosphate myocardial scintigraphy. Semin Nucl Med 1980;10:54-69.

26. Stats MA, Stone JR. Varying levels of small microcalcifications and macrophages in ATTR and AL cardiac amyloidosis: Implications for utilizing nuclear medicine studies to subtype. Cardiovasc Pathol 2016;25:413-7.

27. Van Der Gucht A, Galat A, Rosso J, Guellich A, Garot J, Bodez D, et al. [18F]-NaF PET/CT imaging in cardiac amyloidosis. J Nucl Cardiol 2016;23:846-9.

28. Suhr OB, Lundgren E, Westermark P. One mutation, two distinct disease variants: unravelling the impact of transthyretin amyloid fibril composition. J Intern Med 2017;281:337-47.

29. Pilebro B, Suhr OB, Näslund U, Westermark P, Lindqvist $P$, Sundström T. (99m)Tc-DPD uptake reflects amyloid fibril composition in hereditary transthyretin amyloidosis. Upsala J Med Sci 2016;121:17-24. 